\title{
Non-Existence Results for the Coupled Klein-Gordon-Maxwell Equations
}

\author{
Teresa D'Aprile \\ Dipartimento di Matematica \\ via E. Orabona 4, 70125 BARI, Italy \\ e-mail: daprile@dm.uniba.it \\ Dimitri Mugnai* \\ Dipartimento di Matematica e Informatica \\ via Vanvitelli 1, 06123 PERUGIA, Italy \\ e-mail:mugnai@dipmat.unipg.it \\ Received 16 July 2004 \\ Communicated by Donato Fortunato
}

\begin{abstract}
In this paper we obtain some non-existence results for the Klein-Gordon equation coupled with the electrostatic field. The method relies on the deduction of some suitable Pohožaev identity which provides necessary conditions to get existence of nontrivial solutions. The case of Maxwell-Schrödinger type coupled equations is also considered.

1991 Mathematics Subject Classification. 35B40, 35B45, 35J55.

Key words. Klein-Gordon-Maxwell system, Schrödinger-Maxwell system, non-existence, bound states.
\end{abstract}

\section{Introduction}

This paper deals with non-existence results of nontrivial solutions for some semilinear elliptic systems in $\mathbb{R}^{3}$. Such problems have been motivated by substantial researches generated in recent years concerning certain kinds of solitary charged waves in nonlinear equations of Klein-Gordon or Schrödinger type.

\footnotetext{
*Supported by M.I.U.R. project: Metodi variazionali ed Equazioni Differenziali Nonlineari
} 
More precisely, let us first consider the following system:

$$
\begin{gathered}
-\Delta u+\left[m^{2}-(\omega+e \phi)^{2}\right] u=f(u) \text { in } \mathbb{R}^{3}, \\
-\Delta \phi+e^{2} u^{2} \phi=-e \omega u^{2} \quad \text { in } \mathbb{R}^{3},
\end{gathered}
$$

where $m, \omega, e>0, u, \phi: \mathbb{R}^{3} \rightarrow \mathbb{R}, f: \mathbb{R} \rightarrow \mathbb{R}$. Such system has been first introduced in [2] as a model describing solitary waves for the nonlinear stationary Klein-Gordon equation in the three-dimensional space interacting with the electrostatic field. Here $m$ and $e$ are the mass and the charge of the particle respectively, while $\omega$ denotes the phase. The unknowns of the system are the field $u$ associated to the particle and the electric potential $\phi$. The presence of the nonlinear term simulates the interaction between many particles or external nonlinear perturbations.

Suppose that $f$ is a continuous function such that $f(0)=0$. (1.1) and (1.2) are the Euler-Lagrange equations corresponding to the functional

$$
\mathcal{S}(u, \phi)=\frac{1}{2} \int_{\mathbb{R}^{3}}\left(|\nabla u|^{2}-|\nabla \phi|^{2}+\left[m^{2}-(\omega+e \phi)^{2}\right] u^{2}\right) d x-\int_{\mathbb{R}^{3}} F(u) d x,
$$

where

$$
F(t)=\int_{0}^{t} f(s) d s \quad t \in \mathbb{R} .
$$

For physical reasons, we are led to consider solutions with bounded energy (bound states), i.e. we want the functional $\mathcal{S}$ to be finite. Hence we require $u \in H^{1}$ (i.e. $\left.u,|\nabla u| \in L^{2}\left(\mathbb{R}^{3}\right)\right), \phi \in D^{1}$ (i.e. $\left.|\nabla \phi| \in L^{2}\left(\mathbb{R}^{3}\right)\right)$ and $F(u) \in L^{1}\left(\mathbb{R}^{3}\right)$.

Some existence results for the system (1.1)-(1.2) have been proved in the case $f(u)=|u|^{p-2} u$. In [2] the authors find infinitely many radially symmetric solutions having bounded energy for $4<p<6$; in [10] the range $p \in(2,4]$ is also covered. Motivated by these works, a natural question arises: what happens in the absence of the nonlinear term or if the exponent $p$ varies in different ranges?

In this paper we examine both cases, considering respectively $f \equiv 0$ and $f(u)=$ $|u|^{p-2} u$ with $p \in(0,2] \cup[6,+\infty)$, and we exhibit a negative answer: the unique bound state solution is the trivial one (for more general nonlinear functions $f$, see Theorem 1.2 below).

In order to state the precise result, we first consider the case $f \equiv 0$, which leads to the following system:

$$
\begin{gathered}
-\Delta u+\left[m^{2}-(\omega+e \phi)^{2}\right] u=0 \text { in } \mathbb{R}^{3}, \\
-\Delta \phi+e^{2} u^{2} \phi=-e \omega u^{2} \quad \text { in } \mathbb{R}^{3} .
\end{gathered}
$$

Note that in this case the system describes linear charged Klein-Gordon fields in the presence of the electrostatic field; we point out that equations (1.3)-(1.4) have a relevant physical significance, since they describe a system of isolated charged particles in absence of mutual interactions and without external nonlinear perturbations. However, the nature of the problem is still nonlinear, but the nonlinearity is merely internal to the system, being given only by the coupling, i.e. by the interaction of the particle with its own electrostatic field.

We can now state a first result: 
Theorem 1.1 Assume $m, \omega, e>0$ and let $(u, \phi)$ be a solution of the system (1.3)(1.4) such that $u \in H^{1}$ and $\phi \in D^{1}$. Then $u=\phi=0$.

Concerning the general system (1.1)-(1.2), another consequence of the method employed to prove Theorem 1.1 is the following further nonexistence result.

Theorem 1.2 Let $f: \mathbb{R} \rightarrow \mathbb{R}$ be a continuous function such that $f(0)=0$. Assume $m, \omega, e>0$ and either

i) $f(s) s+2\left(m^{2}-\omega^{2}\right) s^{2} \geq 6 F(s)$ for every $s \in \mathbb{R}$,

or

ii) $2 F(s) \geq f(s) s$ for every $s \in \mathbb{R}$.

Let $(u, \phi)$ be a solution of the system (1.1)-(1.2) such that $u \in H^{1}, \phi \in D^{1}$ and $F(u), f(u) u \in L^{1}\left(\mathbb{R}^{3}\right)$. Then $u=\phi=0$.

If $f(s)=|s|^{p-2} s$ we immediately get the following corollary.

Corollary 1.1 Assume $m, \omega, e>0$ and either

i) $p \geq 6$ and $m \geq \omega$

or

ii) $p \leq 2$.

Let $(u, \phi)$ be a solution of the system (1.1)-(1.2) with $f(s)=|s|^{p-2} s$ such that $u \in H^{1} \cap L^{p}\left(\mathbb{R}^{3}\right)$ and $\phi \in D^{1}$. Then $u=\phi=0$.

Remark 1.1 We point out that the nonexistence result in the critical case $p=6$ was already obtained in [6]. Note that if $p=6$, according to Sobolev embeddings, the requirement $u \in L^{p}\left(\mathbb{R}^{3}\right)$ in corollary 1.1 can be omitted. Moreover, if $p=2$ Theorem 1.2 can be reduced to Theorem 1.1 .

In view of the previous results, the presence of a nonzero nonlinear external perturbation term with a superlinear and subcritical growth seems to be necessary to get nontrivial solitary Klein-Gordon charged waves interacting with their electrostatic field. In the spirit of the method developed in [4]-[5], the idea of the proof is based on a suitable Pohožaev identity ([17]) for the system (1.1)-(1.2) which provides necessary conditions for the existence of nontrivial solutions.

Stationary states of nonlinear Schrödinger equations lead to similar problems. Indeed, in [1] the authors proved that the following system of Maxwell-Schrödinger equations

$$
\begin{gathered}
-\frac{\hbar^{2}}{2 m} \Delta u+\omega u+e \phi u-f(u)=0 \text { in } \mathbb{R}^{3} \\
-\Delta \phi=4 \pi e u^{2} \text { in } \mathbb{R}^{3}
\end{gathered}
$$


actually describes a charged wave interacting with its own electrostatic field. As before, $u$ is the wave associated to the particle, $m, e$ and $\omega$ are the mass, the charge and the phase of the wave respectively, while $\hbar$ is the Planck's constant.

Concerning equations (1.5)-(1.6) with $f(u)=|u|^{p-2} u$, the existence of a nontrivial radial solution was proved in [7] under the restriction $4<p<6$ and in [10] for $4 \leq p<6$, while in [12] the existence of a non-radially symmetric solution was established for $2<p<6$.

We point out that the system (1.5)-(1.6) has attracted considerable attention in recent years: the eigenvalue problem with $f=0$ has been studied in [1] (in the case in which the charged particle lies in a bounded space region $\Omega$ ) and in [8] (in the presence of an external nonzero potential). Furthermore in the papers [9] and [11] the authors deal with the semiclassical limit for the system (1.5)-(1.6), and they find a family of nontrivial solutions exhibiting a concentration behavior when $\hbar \rightarrow 0^{+}$.

In the second part of the paper we prove the following nonexistence results for the system (1.5)-(1.6).

Theorem 1.3 Let $f: \mathbb{R} \rightarrow \mathbb{R}$ be a continuous function with $f(0)=0$. Assume $m, \omega, e>0$ and either

i) $f(s) s+2 \omega s^{2} \geq 6 F(s)$ for every $s \in \mathbb{R}$,

or

ii) $2 F(s) \geq f(s) s$ for every $s \in \mathbb{R}$.

Let $(u, \phi)$ be a solution of the system (1.5)-(1.6) such that $u \in H^{1}, \phi \in D^{1}$ and $F(u), f(u) u \in L^{1}$. Then $u=\phi=0$.

Clearly we have the following corollary.

Corollary 1.2 Assume $m, \omega, e>0$ and either $p \geq 6$ or $p \leq 2$. Let $(u, \phi)$ be a solution of the system (1.5)-(1.6) with $f(s)=|s|^{p-2} s$, such that $u \in H^{1} \cap L^{p}\left(\mathbb{R}^{3}\right)$ and $\phi \in D^{1}$. Then $u=\phi=0$.

As already observed in Remark 1.1, if $p=6$, according to Sobolev embeddings, the requirement $u \in L^{p}\left(\mathbb{R}^{3}\right)$ in corollary 1.2 can be omitted.

Finally we recall that similar physical models of Maxwell-Dirac and KleinGordon-Born-Infeld systems have been studied respectively in [14] and [13], [16]. Moreover in [3] the authors obtain the existence of topological solitary waves interacting with electromagnetic fields.

Let us now briefly outline the organization of the contents of this paper. In section 2 we analyze the variational structure of the system (1.3)-(1.4) and we show that its solutions correspond to the critical points of a $C^{1}$ functional on the space $H^{1}$. Section 3 is devoted to prove the non-existence Theorems 1.1 and 1.2. Finally in section 4 we develop the same arguments to get non-existence results for the Maxwell-Schrödinger type problems (1.5)-(1.6). 


\section{NOTATIONS}

- $L^{p} \equiv L^{p}\left(\mathbb{R}^{3}\right)(1 \leq p<+\infty)$ is the usual Lebesgue space endowed with the norm

$$
\|u\|_{p}^{p}:=\int_{\mathbb{R}^{3}}|u|^{p} d x
$$

- $H^{1} \equiv H^{1}\left(\mathbb{R}^{3}\right)$ denotes the usual Sobolev space endowed with the norm

$$
\|u\|_{H^{1}}^{2}:=\int_{\mathbb{R}^{3}}\left(|\nabla u|^{2}+|u|^{2}\right) d x
$$

- $D^{1} \equiv D^{1}\left(\mathbb{R}^{3}\right)$ is the completion of $C_{0}^{\infty}\left(\mathbb{R}^{3}, \mathbb{R}\right)$ with respect to the norm

$$
\|u\|_{D^{1}}^{2}:=\int_{\mathbb{R}^{3}}|\nabla u|^{2} d x
$$

- we will use the symbol $C$ for denoting positive constants depending only on the functional spaces. The value of $C$ is allowed to change from line to line and also in the same formula.

\section{The Variational setting for the system of Klein- Gordon-Maxwell equations}

In this section we will prove some preliminary results concerning the variational structure for the system (1.3)-(1.4).

First we recall the following continuous embeddings:

$$
H^{1} \hookrightarrow L^{p} \forall p \in[2,6] \quad \text { and } \quad D^{1} \hookrightarrow L^{6} .
$$

We need the following auxiliary Lemma.

Lemma 2.1 For any $u \in H^{1}$ and for any $h \in D^{-1}$ there exists a unique solution $\phi:=\left(\Delta-u^{2}\right)^{-1}[h] \in D^{1}$ of the equation

$$
\Delta \phi-u^{2} \phi=h
$$

(being $D^{-1}$ the dual space of $D^{1}$ ). Moreover, for every $u \in H^{1}$ and for every $h, k \in D^{-1}$,

$$
\left\langle h,\left(\Delta-u^{2}\right)^{-1}[k]\right\rangle=\left\langle k,\left(\Delta-u^{2}\right)^{-1}[h]\right\rangle,
$$

where $\langle\cdot, \cdot\rangle$ denotes the duality pairing between $D^{-1}$ and $D^{1}$.

Proof. The proof concerning the existence part is a straightforward application of Lax-Milgram Lemma. Indeed, if $u \in H^{1}$, then by Hölder's inequality and (2.7),

$$
\int_{\mathbb{R}^{3}} u^{2} \phi^{2} d x \leq C\|u\|_{3}^{2}\|\phi\|_{6}^{2} \leq C\|u\|_{3}^{2}\|\phi\|_{D^{1}}^{2},
$$


and thus $\left(\int|\nabla \phi|^{2}+\int u^{2} \phi^{2}\right)^{1 / 2}$ is a norm in $D^{1}$ equivalent to $\|\phi\|_{D^{1}}$. The remaining part is an easy computation.

A fundamental tool in our analysis will be the following Proposition.

Proposition 2.1 For every $u \in H^{1}$, there exists a unique $\phi=\phi_{u} \in D^{1}$ which solves (1.4), and

$$
-\frac{\omega}{e} \leq \phi_{u} \leq 0 \text { in } \mathbb{R}^{3}
$$

Moreover, the map $\Phi: u \in H^{1} \longrightarrow \phi_{u} \in D^{1}$ is of class $C^{1}$ and for every $u, v \in H^{1}$

$$
\left(\Phi^{\prime}[u]\right)[v]=2 e\left(\Delta-e^{2} u^{2}\right)^{-1}\left[\left(\omega+e \phi_{u}\right) u v\right] .
$$

Proof. The existence and uniqueness part follows from Lemma 2.1, since $u^{2} \in$ $L^{6 / 5} \subset D^{-1}$. See also [2] and [10].

Fixed $u \in H^{1}$, if we multiply $(1.4)$ by $\left(\omega+e \phi_{u}\right)_{-} \equiv-\min \left\{\omega+e \phi_{u}, 0\right\}$, which is an admissible test function, we get

$$
-e \int_{\phi_{u} \leq-\omega / e}\left|\nabla \phi_{u}\right|^{2} d x-e \int_{\phi_{u} \leq-\omega / e}\left(\omega+e \phi_{u}\right)^{2} u^{2} d x=0
$$

which implies $\phi_{u} \geq-\frac{\omega}{e}$. Next use $\left(\phi_{u}\right)_{+}=\max \left\{\phi_{u}, 0\right\}$ as a test function in (1.4) to get

$$
\int_{\phi_{u} \geq 0}\left|\nabla \phi_{u}\right|^{2} d x+e \int_{\phi_{u} \geq 0}\left(\omega+e \phi_{u}\right) \phi_{u} u^{2} d x=0,
$$

by which, since $\omega+e \phi_{u} \geq 0,\left(\phi_{u}\right)_{+} \equiv 0$.

For the last part, consider the map $T: H^{1} \times D^{1} \rightarrow D^{1}$ of class $C^{1}$

$$
T(u, \phi)=e \Delta^{-1}\left[(\omega+e \phi) u^{2}\right]-\phi .
$$

Note that $T$ is well defined, since by $(2.7) u^{2}, u^{2} \phi \in L^{6 / 5} \subset D^{-1}$. It is obvious that $(u, \phi)$ solves (1.4) if and only if $T(u, \phi)=0$.

Now, for every $(u, \phi) \in H^{1} \times D^{1}$ we compute

$$
\frac{\partial T}{\partial \phi}(u, \phi): D^{1} \rightarrow D^{1}, \quad \psi \mapsto e^{2} \Delta^{-1}\left[u^{2} \psi\right]-\psi
$$

and

$$
\frac{\partial T}{\partial u}(u, \phi): H^{1} \rightarrow D^{1}, \quad v \mapsto 2 e \Delta^{-1}[(\omega+e \phi) u v] .
$$

It immediately follows that $\frac{\partial T}{\partial \phi}(u, \phi)$ is invertible for every $(u, \phi) \in H^{1} \times D^{1}$ and

$$
\left(\frac{\partial T}{\partial \phi}(u, \phi)\right)^{-1}=\left(e^{2} u^{2}-\Delta\right)^{-1} \circ \Delta .
$$

Then the $C^{1}$ regularity of the map $\Phi$ follows from the implicit function theorem, and, for every $u \in H^{1}, \Phi^{\prime}[u]: H^{1} \rightarrow D^{1}$ is given by (2.10). 
Now let us consider the functional $J: H^{1} \longrightarrow \mathbb{R}$ defined as

$$
J(u)=\frac{1}{2} \int_{\mathbb{R}^{3}}|\nabla u|^{2} d x+\frac{m^{2}-\omega^{2}}{2} \int_{\mathbb{R}^{3}} u^{2} d x-\frac{e \omega}{2} \int_{\mathbb{R}^{3}} u^{2} \phi_{u} d x .
$$

The next lemma establishes the variational nature of the system (1.3)-(1.4).

Lemma 2.2 The following statements are equivalent:

i) $(u, \phi) \in H^{1} \times D^{1}$ is a solution of the system (1.3)-(1.4);

ii) $u \in H^{1}$ is a critical point of $J$ and $\phi=\phi_{u}$.

Proof. By (2.10), for every $u, v \in H^{1}$ we have

$$
\begin{aligned}
J^{\prime}(u)[v]= & \int_{\mathbb{R}^{3}} \nabla u \cdot \nabla v d x+\left(m^{2}-\omega^{2}\right) \int_{\mathbb{R}^{3}} u v d x-e \omega \int_{\mathbb{R}^{3}} u v \phi_{u} d x \\
& -e^{2} \omega \int_{\mathbb{R}^{3}} u^{2}\left(\Delta-e^{2} u^{2}\right)^{-1}\left[\left(\omega+e \phi_{u}\right) u v\right] d x \\
= & \int_{\mathbb{R}^{3}} \nabla u \cdot \nabla v d x+\left(m^{2}-\omega^{2}\right) \int_{\mathbb{R}^{3}} u v d x-e \omega \int_{\mathbb{R}^{3}} u v \phi_{u} d x \\
& -e \int_{\mathbb{R}^{3}}\left(\Delta-e^{2} u^{2}\right)^{-1}\left[e \omega u^{2}\right]\left(\omega+e \phi_{u}\right) u v d x
\end{aligned}
$$

by (2.8). On the other hand, in any case we know that $\left(\Delta-e^{2} u^{2}\right)^{-1}\left[\omega e u^{2}\right]=\phi_{u}$, by which

$$
J^{\prime}(u)[v]=\int_{\mathbb{R}^{3}} \nabla u \cdot \nabla v d x+\left(m^{2}-\omega^{2}\right) \int_{\mathbb{R}^{3}} u v d x-e \int_{\mathbb{R}^{3}}\left(2 \omega+e \phi_{u}\right) \phi_{u} u v d x
$$

and the thesis follows.

\section{Nonexistence results for the system of Klein- Gordon-Maxwell equations}

In this Section we prove Theorems 1.1 and 1.2 as a corollary of a suitable Pohožaevtype identity for the systems (1.1)-(1.2) and (1.3)-(1.4).

First we establish the following identities.

Lemma 3.1 Let $u, \phi \in H_{\text {loc }}^{2}\left(\mathbb{R}^{N}\right)$ and $a, b \geq 0$. Then, for every $R>0$, the following identities hold:

$$
\begin{aligned}
\int_{B_{R}}-\Delta u(x \cdot \nabla u) d x= & \frac{2-N}{2} \int_{B_{R}}|\nabla u|^{2} d x-\frac{1}{R} \int_{\partial B_{R}}|x \cdot \nabla u|^{2} d \sigma \\
& +\frac{R}{2} \int_{\partial B_{R}}|\nabla u|^{2} d \sigma ;
\end{aligned}
$$




$$
\begin{aligned}
\int_{B_{R}}(a+b \phi) \phi u(x \cdot \nabla u) d x & =-\int_{B_{R}}\left(\frac{a}{2}+b \phi\right) u^{2}(x \cdot \nabla \phi) d x \\
- & \frac{N}{2} \int_{B_{R}}(a+b \phi) \phi u^{2} d x+\frac{R}{2} \int_{\partial B_{R}}(a+b \phi) \phi u^{2} d \sigma ; \\
\int_{B_{R}} g(u)(x \cdot \nabla u) d x & =-N \int_{B_{R}} G(u) d x+R \int_{\partial B_{R}} G(u) d \sigma,
\end{aligned}
$$

where $g: \mathbb{R} \rightarrow \mathbb{R}$ is a continuous function such that $g(0)=0$ and $G(s)=\int_{0}^{s} g(t) d t$.

Proof. The proof of (3.14) can be found in [4, Proof of Proposition 1, pg. 320]. Concerning (3.12), fix $i, j=1, \ldots, N$ and, integrating by parts on a ball $B_{R}$, we compute

$$
\begin{aligned}
\int_{B_{R}} u_{x_{j}} u_{x_{i} x_{j}} x_{i} d x & =\frac{1}{2} \int_{B_{R}}\left(\left|u_{x_{j}}\right|^{2}\right)_{x_{i}} x_{i} d x \\
& =-\frac{1}{2} \int_{B_{R}}\left|u_{x_{j}}\right|^{2} d x+\frac{1}{2} \int_{\partial B_{R}}\left|u_{x_{j}}\right|^{2} \frac{\left|x_{i}\right|^{2}}{|x|} d \sigma .
\end{aligned}
$$

Therefore, denoting by $\delta_{i j}$ the Kroneker symbols,

$$
\begin{aligned}
-\int_{B_{R}} u_{x_{j} x_{j}} & u_{x_{i}} x_{i} d x=\int_{B_{R}} u_{x_{j}} u_{x_{i} x_{j}} x_{i} d x+\int_{B_{R}} u_{x_{j}} u_{x_{i}} \delta_{i j} d x \\
& -\int_{\partial B_{R}} u_{x_{j}} u_{x_{i}} \frac{x_{i} x_{j}}{|x|} d \sigma=-\frac{1}{2} \int_{B_{R}}\left|u_{x_{j}}\right|^{2} d x+\int_{B_{R}} u_{x_{j}} u_{x_{i}} \delta_{i j} d x \\
& +\frac{1}{2} \int_{\partial B_{R}}\left|u_{x_{j}}\right|^{\mid} \frac{\left|x_{i}\right|^{2}}{|x|} d \sigma-\int_{\partial B_{R}} u_{x_{j}} u_{x_{i}} \frac{x_{i} x_{j}}{|x|} d \sigma .
\end{aligned}
$$

Summing up for $i, j=1, \ldots, N,(3.12)$ follows.

In order to prove (3.13), fix $i=1, \ldots, N$ and, integrating by parts, compute

$$
\begin{aligned}
& 2 \int_{B_{R}}(a+b \phi) \phi u u_{x_{i}} x_{i} d x=\int_{B_{R}}(a+b \phi) \phi\left(u^{2}\right)_{x_{i}} x_{i} d x \\
& \quad=-\int_{B_{R}}(a+2 b \phi) \phi_{x_{i}} u^{2} x_{i} d x-\int_{B_{R}}(a+b \phi) \phi u^{2} d x+\int_{\partial B_{R}}(a+b \phi) \phi u^{2} \frac{x_{i}^{2}}{|x|} d \sigma .
\end{aligned}
$$

Summing up for $i=1, \ldots, N$, we get the thesis.

Now we are able to prove Theorem 1.1.

Proof of Theorem 1.1. Let $(u, \phi) \in H^{1} \times D^{1}$ be a solution to (1.3)-(1.4).

First let us remark that, if $m \geq \omega$, the proof is straightforward: indeed, if we multiply equation (1.3) by $u$ and integrate by parts, we obtain

$$
\int_{\mathbb{R}^{3}}|\nabla u|^{2} d x+\int_{\mathbb{R}^{3}}\left[m^{2}-(\omega+e \phi)^{2}\right] u^{2} d x=0
$$

and then $u \equiv 0$ by $(2.9)$, since $m^{2}-(\omega+e \phi)^{2} \geq m^{2}-\omega^{2} \geq 0$. 
For the general case, let us recall that $u, \phi \in H_{l o c}^{2}\left(\mathbb{R}^{3}\right)$ by standard regularity results; then equations (1.3)-(1.4) are verified a.e. in $\mathbb{R}^{3}$. Set $\Omega=m^{2}-\omega^{2}$ and multiply (1.3) by $x \cdot \nabla u$; integrating on $B_{R}$ and using Lemma 3.1 with $g(s)=s$, we deduce

$$
\begin{aligned}
& -\frac{1}{2} \int_{B_{R}}|\nabla u|^{2} d x-\frac{3}{2} \Omega \int_{B_{R}} u^{2} d x+e \int_{B_{R}}(\omega+e \phi) u^{2}(x \cdot \nabla \phi) d x \\
& +\frac{3}{2} e \int_{B_{R}}(2 \omega+e \phi) \phi u^{2} d x=\frac{1}{R} \int_{\partial B_{R}}|x \cdot \nabla u|^{2} d \sigma \\
& -\frac{R}{2} \int_{\partial B_{R}}|\nabla u|^{2} d \sigma-\frac{\Omega}{2} R \int_{\partial B_{R}} u^{2} d \sigma+\frac{R}{2} e \int_{\partial B_{R}}(2 \omega+e \phi) \phi u^{2} d \sigma .
\end{aligned}
$$

Now multiply (1.4) by $x \cdot \nabla \phi$; integrating on $B_{R}$ and using again Lemma 3.1 , we achieve

$$
\begin{aligned}
& e \int_{B_{R}}(\omega+e \phi) u^{2}(x \cdot \nabla \phi) d x=\int_{B_{R}} \Delta \phi(x \cdot \nabla \phi) d x \\
& \quad=\frac{1}{2} \int_{B_{R}}|\nabla \phi|^{2} d x+\frac{1}{R} \int_{\partial B_{R}}|x \cdot \nabla \phi|^{2} d \sigma-\frac{R}{2} \int_{\partial B_{R}}|\nabla \phi|^{2} d \sigma .
\end{aligned}
$$

Combining (3.16) and (3.17) we obtain

$$
\begin{aligned}
- & \frac{1}{2} \int_{B_{R}}\left(|\nabla u|^{2}-|\nabla \phi|^{2}\right) d x-\frac{3}{2} \Omega \int_{B_{R}} u^{2} d x+\frac{3}{2} e \int_{B_{R}}(2 \omega+e \phi) \phi u^{2} d x \\
= & \frac{1}{R} \int_{\partial B_{R}}\left(|x \cdot \nabla u|^{2}-|x \cdot \nabla \phi|^{2}\right) d \sigma-\frac{R}{2} \int_{\partial B_{R}}\left(|\nabla u|^{2}-|\nabla \phi|^{2}\right) d \sigma \\
& -\frac{\Omega}{2} R \int_{\partial B_{R}} u^{2} d \sigma+\frac{R}{2} e \int_{\partial B_{R}}(2 \omega+e \phi) \phi u^{2} d \sigma .
\end{aligned}
$$

Following the idea in [4], we will show that the right hand side in (3.18) converges to zero for a suitable sequence $R_{n} \rightarrow+\infty$. First note how, assuming this, the thesis easily follows. Indeed, considering the identity (3.18) with $R=R_{n}$ and letting $n \rightarrow \infty$, we obtain

$$
-\int_{\mathbb{R}^{3}}\left(|\nabla u|^{2}-|\nabla \phi|^{2}\right) d x-3 \Omega \int_{\mathbb{R}^{3}} u^{2} d x+3 e \int_{\mathbb{R}^{3}}(2 \omega+e \phi) \phi u^{2} d x=0 .
$$

On the other hand, by (1.3) and (1.4) we deduce respectively

$$
\begin{gathered}
\Omega \int_{\mathbb{R}^{3}}|u|^{2} d x=-\int_{\mathbb{R}^{3}}|\nabla u|^{2} d x+e \int_{\mathbb{R}^{3}}(2 \omega+e \phi) \phi u^{2} d x \\
\int_{\mathbb{R}^{3}}|\nabla \phi|^{2} d x=-e \int_{\mathbb{R}^{3}}(\omega+e \phi) \phi u^{2} d x
\end{gathered}
$$

Inserting (3.20) and (3.21) in (3.19), we get

$$
2 \int_{\mathbb{R}^{3}}|\nabla u|^{2} d x-e \int_{\mathbb{R}^{3}}(\omega+e \phi) \phi u^{2} d x=0 .
$$


By $(2.9)(\omega+e \phi) \phi \leq 0$; then $u=0$ and, consequently, $\phi=0$.

Thus it remains to prove that the right hand side of (3.18) goes to zero for a suitable $R_{n} \rightarrow+\infty$. To this aim first note that $|x \cdot \nabla u| \leq R|\nabla u|$ and $|x \cdot \nabla \phi| \leq R|\nabla \phi|$ on $\partial B_{R}$, and, by Proposition 2.1, $|(2 \omega+e \phi) \phi| u^{2} \leq 2 \frac{\omega^{2}}{e} u^{2}$ in $\mathbb{R}^{3}$. Hence it is sufficient to prove that if $\alpha \in L^{1} \cap H_{l o c}^{1}\left(\mathbb{R}^{3}\right)$, then

$$
R_{n} \int_{\partial B_{R_{n}}}|\alpha| d \sigma \rightarrow 0
$$

for a suitable $R_{n} \rightarrow \infty$. Otherwise there would exist $c, R_{0}>0$ such that

$$
\int_{\partial B_{R}}|\alpha| d \sigma \geq \frac{c}{R} \quad \text { for } R \geq R_{0}
$$

and this leads to a contradiction, since

$$
\int_{\mathbb{R}^{3}}|\alpha| d x=\int_{0}^{+\infty} d R \int_{\partial B_{R}}|\alpha| d \sigma \geq c \int_{R_{0}}^{+\infty} \frac{d R}{R}=+\infty .
$$

Remark 3.1 We recall that the solutions of the system (1.3)-(1.4) are the couples $\left(u, \phi_{u}\right)$, where $u$ is a critical point for the functional $J$ defined in $(2.11)$ and $\phi_{u}$ has been defined in Proposition 2.1. We point out that the non-existence result of Theorem 1.1 could be obtained directly for the solutions $u$ which are local minimum point for $J$. Indeed, if $u$ is a local minimum point of $J$, for $\lambda>0$ consider the functions

$$
u_{\lambda}(x)=\lambda u(\lambda x) .
$$

It is easy to show that $\left(u_{\lambda}(x), \phi_{u}(\lambda x)\right)$ solves equation (1.4), hence by the uniqueness given in Proposition 2.1, we immediately get

$$
\phi_{u_{\lambda}}(x)=\phi_{u}(\lambda x)
$$

Now set $a_{u}(\lambda)=J\left(u_{\lambda}\right)$. Taking into account of (2.11), an easy computation gives

$$
a_{u}(\lambda)=\frac{\lambda}{2} \int_{\mathbb{R}^{3}}|\nabla u|^{2} d x+\frac{m^{2}-\omega^{2}}{2 \lambda} \int_{\mathbb{R}^{3}} u^{2} d x-\frac{e \omega}{2 \lambda} \int_{\mathbb{R}^{3}} u^{2} \phi_{u} d x
$$

Since $\lambda=1$ is a local minimum for $a_{u}$, then, by differentiation,

$$
\int_{\mathbb{R}^{3}}|\nabla u|^{2} d x-\left(m^{2}-\omega^{2}\right) \int_{\mathbb{R}^{3}} u^{2} d x+e \omega \int_{\mathbb{R}^{3}} u^{2} \phi_{u} d x=0 .
$$

Substituting (3.20) in (3.23) we obtain (3.22) and we can conclude as in Theorem 1.1 .

The preceding argument ceases to work, however, for generic solutions. Indeed, if $u$ is a local minimum point for $J$, then $\lambda=1$ is obviously a local minimum for $a_{u}$ 
as well (and, consequently, $a_{u}^{\prime}(1)=0$ ). On the contrary, in the generic case, if $u$ is critical for $J$, the conclusion that $\lambda=1$ is critical for $a_{u}$ does not hold in general; to conclude this, one should show that the curve $\lambda>0 \mapsto u_{\lambda} \in H^{1}$ is differentiable at $\lambda=1$, i.e. $\frac{d}{d \lambda} u_{\lambda}(x)=x \cdot \nabla u$ lies in $H^{1}$. If some a priori decays on $u$ and $\nabla u$ are known, we can use them to obtain a simpler proof of the identity (3.22), by making the preceding scale-change argument rigorous. However, the proof of Theorem 1.1 we gave above relies essentially on the Pohožaev identity and provides a rigorous proof of the fact that any solution of the system (1.3)-(1.4) satisfies the identity (3.22).

We conclude this section with the proof of the more general non-existence result given by Theorem 1.2.

Proof of Theorem 1.2. By repeating the same proof of Theorem 1.1, we set $\Omega=$ $m^{2}-\omega^{2}$ and multiply (1.1) by $x \cdot \nabla u$; integrating on $B_{R}$ and using Lemma 3.1 with $g(s)=s$ and $g(s)=f(s)$, we obtain the analogous of (3.18):

$$
\begin{aligned}
- & \frac{1}{2} \int_{B_{R}}\left(|\nabla u|^{2}-|\nabla \phi|^{2}\right) d x-\frac{3}{2} \Omega \int_{B_{R}} u^{2} d x+\frac{3}{2} e \int_{B_{R}}(2 \omega+e \phi) \phi u^{2} d x \\
& +3 \int_{B_{R}} F(u) d x \\
= & \frac{1}{R} \int_{\partial B_{R}}\left(|x \cdot \nabla u|^{2}-|x \cdot \nabla \phi|^{2}\right) d \sigma-\frac{R}{2} \int_{\partial B_{R}}\left(|\nabla u|^{2}-|\nabla \phi|^{2}\right) d \sigma \\
& -\frac{\Omega}{2} R \int_{\partial B_{R}} u^{2} d \sigma+\frac{R}{2} e \int_{\partial B_{R}}(2 \omega+e \phi) \phi u^{2} d \sigma+R \int_{\partial B_{R}} F(u) d \sigma .
\end{aligned}
$$

As already done in the proof of Theorem 1.1, we can find a sequence $R_{n} \rightarrow+\infty$ such that the left hand side of (3.24) vanishes. Hence we are reduced to

$$
\begin{aligned}
& -\frac{1}{2} \int_{\mathbb{R}^{3}}\left(|\nabla u|^{2}-|\nabla \phi|^{2}\right) d x-\frac{3}{2} \Omega \int_{\mathbb{R}^{3}} u^{2} d x+\frac{3}{2} e \int_{\mathbb{R}^{3}}(2 \omega+e \phi) \phi u^{2} d x \\
& \quad+3 \int_{\mathbb{R}^{3}} F(u) d x=0 .
\end{aligned}
$$

By substituting (3.21) we get

$$
-\int_{\mathbb{R}^{3}}|\nabla u|^{2} d x-3 \Omega \int_{\mathbb{R}^{3}} u^{2} d x+e \int_{\mathbb{R}^{3}}(5 \omega+2 e \phi) \phi u^{2} d x+6 \int_{\mathbb{R}^{3}} F(u) d x=0 .
$$

From (1.1) we obtain

$$
\int_{\mathbb{R}^{3}}|\nabla u|^{2} d x+\Omega \int_{\mathbb{R}^{3}} u^{2} d x-e \int_{\mathbb{R}^{3}}(2 \omega+e \phi) \phi u^{2} d x-\int_{\mathbb{R}^{3}} f(u) u d x=0 .
$$

We first isolate $\int|\nabla u|^{2}$ in (3.26) and substitute it in (3.25) to achieve

$$
-2 \Omega \int_{\mathbb{R}^{3}} u^{2} d x+e \int_{\mathbb{R}^{3}}(3 \omega+e \phi) \phi u^{2} d x+\int_{\mathbb{R}^{3}}(6 F(u)-f(u) u) d x=0 .
$$


Since $(3 \omega+e \phi) \phi \leq 0$ by Proposition 2.1, if $6 F(s)-f(s) s-2 \Omega s^{2} \leq 0$ for every $s$, then (3.27) gives $u=0$.

Now we isolate $\Omega \int u^{2}$ in (3.26) and and insert it in (3.25). We end up with

$$
2 \int_{\mathbb{R}^{3}}|\nabla u|^{2} d x-e \int_{\mathbb{R}^{3}}(\omega+e \phi) \phi u^{2} d x+3 \int_{\mathbb{R}^{3}}(2 F(u)-f(u) u) d x=0 .
$$

If $2 F(s)-f(s) s \geq 0$ for every $s$, identity (3.28) and again Proposition 2.1 give $u=0$.

Remark 3.2 Assume that there exist $c_{1}, c_{2}>0$ and $p>1$ such that

$$
|f(s)| \leq c_{1}|s|+c_{2}|s|^{p-1} \quad \forall s \in \mathbb{R},
$$

so that the Nemitsky operator $u \in H^{1} \cap L^{p} \mapsto F(u) \in L^{1}$ is of class $C^{1}$. Then, by repeating the same proof of Lemma 2.2, we deduce that the solutions of the system (1.1)-(1.2) correspond to the critical points of the $C^{1}$ functional

$$
\tilde{J}(u)=\frac{1}{2} \int_{\mathbb{R}^{3}}|\nabla u|^{2} d x+\frac{m^{2}-\omega^{2}}{2} \int_{\mathbb{R}^{3}} u^{2} d x-\frac{e \omega}{2} \int_{\mathbb{R}^{3}} u^{2} \phi_{u} d x-\int_{\mathbb{R}^{3}} F(u) d x .
$$

The non-existence of nontrivial local minima for $\tilde{J}$ can be obtained in a simpler way proceeding as in Remark 3.1. Indeed, considering the real function

$$
\tilde{a}(\lambda)=\tilde{J}\left(u_{\lambda}\right),
$$

where $u_{\lambda}(x)=\lambda u(\lambda x)$, then imposing $\tilde{a}^{\prime}(1)=0$, we immediately arrive at (3.25). The rest follows as above.

\section{Nonexistence results for the System of Maxwell- Schrödinger equations}

As a consequence of the method introduced in the previous section, we are now able to prove some non-existence results for the system (1.5)-(1.6) of coupled SchrödingerMaxwell equations. In this case the variational structure is easier to analyze since we have an explicit representation formula for the solution of equation (1.6), as stated in the following Lemma.

Proposition 4.1 For every $u \in H^{1}$ there exists a unique $\phi=\phi_{u} \in D^{1}$ which solves equation (1.6). Furthermore, $\phi_{u}$ is given by

$$
\phi_{u}(x)=e \int_{\mathbb{R}^{3}} \frac{1}{|x-y|} u^{2}(y) d y .
$$

As a consequence, the map $\Phi: u \in H^{1} \longrightarrow \phi_{u} \in D^{1}$ is of class $C^{1}$ and

$$
(\Phi[u])^{\prime}[v](x)=2 e \int_{\mathbb{R}^{3}} \frac{1}{|x-y|} u(y) v(y) d y \quad \forall u, v \in H^{1} .
$$


Proof. The existence and uniqueness part follows by Lemma 2.1, since $u^{2} \in L^{6 / 5} \subset$ $D^{-1}$. The representation formula holds for $u \in C_{0}^{\infty}\left(\mathbb{R}^{3}\right)$ (for example, see $[15$, Theorem 1, p. 23]); by density it can be extended for any $u \in H^{1}$.

In view of the previous Proposition, the energy functional associated to (1.5)(1.6) has the form

$$
I(u)=\frac{\hbar^{2}}{4 m} \int_{\mathbb{R}^{3}}|\nabla u|^{2} d x+\frac{\omega}{2} \int_{\mathbb{R}^{3}} u^{2} d x+\frac{e}{4} \int_{\mathbb{R}^{3}} u^{2} \phi_{u} d x-\int_{\mathbb{R}^{3}} F(u) d x .
$$

Lemma 4.1 Assume that there exist $c_{1}, c_{2}>0$ and $p>1$ such that

$$
|f(s)| \leq c_{1}|s|+c_{2}|s|^{p-1} \quad \forall s \in \mathbb{R}
$$

Then the following statements are equivalent:

i) $(u, \phi) \in\left(H^{1} \cap L^{p}\right) \times D^{1}$ is a solution of the system (1.3)-(1.4);

ii) $u \in H^{1} \cap L^{p}$ is a critical point of $I$ and $\phi=\phi_{u}$.

Proof. By the assumption (4.30), the Nemitsky operator $u \in H^{1} \cap L^{p} \mapsto F(u) \in L^{1}$ is of class $C^{1}$. Hence, by Proposition 4.1, for every $u, v \in H^{1}$

$$
\begin{aligned}
I^{\prime}(u)[v]= & \frac{\hbar^{2}}{2 m} \int_{\mathbb{R}^{3}} \nabla u \cdot \nabla v d x+\omega \int_{\mathbb{R}^{3}} u v d x \\
& +\frac{e^{2}}{2} \int_{\mathbb{R}^{3}} u(x) v(x) d x \int_{\mathbb{R}^{3}} \frac{1}{|x-y|} u^{2}(y) d y \\
& +\frac{e^{2}}{2} \int_{\mathbb{R}^{3}} u^{2}(x) \int_{\mathbb{R}^{3}} \frac{1}{|x-y|} u(y) v(y) d y-\int_{\mathbb{R}^{3}} f(u) v d x \\
= & \frac{\hbar^{2}}{2 m} \int_{\mathbb{R}^{3}} \nabla u \cdot \nabla v d x+\omega \int_{\mathbb{R}^{3}} u v d x-e \int_{\mathbb{R}^{3}} u v \phi_{u} d x-\int_{\mathbb{R}^{3}} f(u) v d x
\end{aligned}
$$

by Fubini-Tonelli's Theorem, and the conclusion follows.

We conclude this section with the proof of the non-existence result given by Theorem 1.3.

Proof of Theorem 1.3. For the sake of simplicity, set $\hbar=2 m=1$. Following the proof of Theorem 1.1, multiplying (1.5) by $x \cdot \nabla u$, integrating on $B_{R}$ and using Lemma 3.1 with $g(s)=s$ and $g(s)=f(s)$, we obtain:

$$
\begin{aligned}
& -\frac{1}{2} \int_{B_{R}}|\nabla u|^{2} d x-\frac{3}{2} \omega \int_{B_{R}} u^{2} d x-\frac{e}{2} \int_{B_{R}} u^{2}(x \cdot \nabla \phi) d x-\frac{3}{2} e \int_{B_{R}} \phi u^{2} d x \\
& +3 \int_{B_{R}} F(u) d x=\frac{1}{R} \int_{\partial B_{R}}|x \cdot \nabla u|^{2} d \sigma-\frac{R}{2} \int_{\partial B_{R}}|\nabla u|^{2} d \sigma \\
& -\frac{\omega}{2} R \int_{\partial B_{R}} u^{2} d \sigma-\frac{R}{2} e \int_{\partial B_{R}} \phi u^{2} d \sigma+R \int_{\partial B_{R}} F(u) d \sigma .
\end{aligned}
$$


Now multiply (1.6) by $x \cdot \nabla \phi$; integrating on $B_{R}$ and using again Lemma 3.1, we achieve

$$
\begin{aligned}
& 4 \pi e \int_{B_{R}} u^{2}(x \cdot \nabla \phi) d x=\int_{B_{R}}-\Delta \phi(x \cdot \nabla \phi) d x \\
& =-\frac{1}{2} \int_{B_{R}}|\nabla \phi|^{2} d x-\frac{1}{R} \int_{\partial B_{R}}|x \cdot \nabla \phi|^{2} d \sigma+\frac{R}{2} \int_{\partial B_{R}}|\nabla \phi|^{2} d \sigma .
\end{aligned}
$$

Combining (4.31) and (4.32) we obtain

$$
\begin{aligned}
& -\frac{1}{2} \int_{B_{R}}\left(|\nabla u|^{2}-\frac{1}{8 \pi}|\nabla \phi|^{2}\right) d x-\frac{3}{2} \omega \int_{B_{R}} u^{2} d x-\frac{3}{2} e \int_{B_{R}} \phi u^{2} d x \\
& +3 \int_{B_{R}} F(u) d x=\frac{1}{R} \int_{\partial B_{R}}\left(|x \cdot \nabla u|^{2}-\frac{1}{8 \pi}|x \cdot \nabla \phi|^{2}\right) d \sigma \\
& -\frac{R}{2} \int_{\partial B_{R}}\left(|\nabla u|^{2}-\frac{1}{8 \pi}|\nabla \phi|^{2}\right) d \sigma \\
& -\frac{\omega}{2} R \int_{\partial B_{R}} u^{2} d \sigma-\frac{R}{2} e \int_{\partial B_{R}} \phi u^{2} d \sigma+R \int_{\partial B_{R}} F(u) d \sigma .
\end{aligned}
$$

As already done in the proof of Theorem 1.1, we can find a sequence $R_{n} \rightarrow+\infty$ such that the right hand side of (4.33) vanishes. Hence we are reduced to

$$
\int_{\mathbb{R}^{3}}\left(|\nabla u|^{2}-\frac{1}{8 \pi}|\nabla \phi|^{2}\right) d x+3 \omega \int_{\mathbb{R}^{3}} u^{2} d x+3 e \int_{\mathbb{R}^{3}} \phi u^{2} d x-6 \int_{\mathbb{R}^{3}} F(u) d x=0 .
$$

By (1.6), we deduce

$$
\int_{\mathbb{R}^{3}}|\nabla \phi|^{2} d x=4 \pi e \int_{\mathbb{R}^{3}} \phi u^{2} d x
$$

By substituting in (4.34) we get

$$
-2 \int_{\mathbb{R}^{3}}|\nabla u|^{2} d x-6 \omega \int_{\mathbb{R}^{3}} u^{2} d x-5 e \int_{\mathbb{R}^{3}} \phi u^{2} d x+12 \int_{\mathbb{R}^{3}} F(u) d x=0 .
$$

From (1.5) we obtain

$$
\int_{\mathbb{R}^{3}}|\nabla u|^{2} d x+\omega \int_{\mathbb{R}^{3}} u^{2} d x+e \int_{\mathbb{R}^{3}} \phi u^{2} d x-\int_{\mathbb{R}^{3}} f(u) u d x=0 .
$$

We first isolate $\int|\nabla u|^{2}$ in (4.36) and substitute it in (4.35) to achieve

$$
-4 \omega \int_{\mathbb{R}^{3}} u^{2} d x-3 e \int_{\mathbb{R}^{3}} \phi u^{2} d x+2 \int_{\mathbb{R}^{3}}(6 F(u)-f(u) u) d x=0 .
$$

By Proposition 4.1, $\phi=\phi_{u} \geq 0$. Thus, if $6 F(s)-f(s) s-2 \omega s^{2} \leq 0$ for every $s$, then (4.37) gives $u=0$.

Now we isolate $\omega \int u^{2}$ in (4.36) and we insert it in (4.35). We end up with

$$
4 \int_{\mathbb{R}^{3}}|\nabla u|^{2} d x+e \int_{\mathbb{R}^{3}} \phi u^{2} d x+6 \int_{\mathbb{R}^{3}}(2 F(u)-f(u) u) d x=0 .
$$


If $2 F(u)-f(u) u \geq 0$ for every $s$, identity (4.38) and again Proposition 4.1 give $u=0$.

Remark 4.1 Assume that $f$ satisfies (4.30). Then, according to Lemma 4.1, the solutions of the system (1.5)-(1.6) correspond to the critical points of the $C^{1}$ functional $I$ defined in (4.29). The non-existence of nontrivial local minima $u$ for $I$ can be obtained in a simpler way proceeding as in Remark 3.1 by a scale change argument. Indeed, consider the real function

$$
b(\lambda)=I\left(u_{\lambda}\right),
$$

where $u_{\lambda}(x)=u(\lambda x)$. Then, Lemma 4.1 gives $\phi_{u_{\lambda}}(x)=\lambda^{-2} \phi_{u}(\lambda x)$, and imposing $b^{\prime}(1)=0$, we immediately arrive at (4.35). The rest follows as above.

Remark 4.2 By multiplying both members of (1.5) by $u$ and integrating by parts, we immediately obtain that the presence of a nonlinear term is necessary to get existence of nontrivial solutions for the system (1.5)-(1.6). On the other hand, it is easy to prove that, for every $2<p<6$, there exists at least a radially symmetric solution of (1.5)-(1.6) with $f(u)=\gamma|u|^{p-2} u$ for a suitable $\gamma>0$. Indeed, it is sufficient to minimize the functional

$$
T(u)=\frac{\hbar^{2}}{4 m} \int_{\mathbb{R}^{3}}|\nabla u|^{2} d x+\frac{\omega}{2} \int_{\mathbb{R}^{3}} u v d x+\frac{e^{2}}{4} \int_{\mathbb{R}^{3}} u^{2}(x) d x \int_{\mathbb{R}^{3}} \frac{1}{|x-y|} u^{2}(y) d y
$$

over the manifold

$$
\mathcal{M}:=\left\{\left.u \in H_{r}^{1}\left|\int_{\mathbb{R}^{3}}\right| u\right|^{p} d x=1\right\},
$$

where $H_{r}^{1}$ denotes the subspace of $H^{1}$ made up of the radially symmetric functions. According to the compact injection $H_{r}^{1} \hookrightarrow L^{p}$ (see [4, Theorem A.I', pg. 341] or [18]) $\mathcal{M}$ is a compact manifold, while $T$ is weakly lower semicontinuous on $H_{r}^{1}$. Hence we easily get the existence of a minimizing function. The constraint causes a Lagrange multiplier to appear and one obtains a positive solution $u$ of $-\frac{\hbar^{2}}{2 m} \Delta u+\omega u+e u \phi_{u}=$ $\gamma|u|^{p-2} u$. However, the Lagrange multiplier $\gamma$ cannot be removed by looking for a solution of the form $v=\sigma u$ because of the different features of homogeneity of the terms of $T$.

Acknowledgement This paper was carried out while the authors were visiting Professor Charles Stuart at the Centre Bernoulli at the "École Polytechnique Fédérale de Lausanne". The authors are very grateful to C. Stuart for stimulating discussions and to all the staff of Centre Bernoulli for their warm hospitality.

\section{References}

[1] V. Benci and D. Fortunato, An eigenvalue problem for the Schrödinger-Maxwell equations, Top. Meth. Nonlinear Anal. 11 (1998), 283-293. 
[2] V. Benci and D. Fortunato, Solitary waves of the nonlinear Klein-Gordon equation coupled with the Maxwell equations, Rev. Math. Phys. 14 (2002), 409-420.

[3] V. Benci, D. Fortunato, A. Masiello and L. Pisani, Solitons and electromagnetic fields, Math. Z. 232 (1999), 73-102.

[4] H. Berestycki and P.L. Lions, Nonlinear scalar field equatios, I- Existence of a ground state, Arch. Rat. Mech. Anal. 82 (1983), no. 4, 313-345.

[5] H. Berestycki and P.L. Lions, Nonlinear scalar field equatios, II - Existence of infinitely many solutions, Arch. Rat. Mech. Anal. 82 (1983), no. 4, 347-375.

[6] D. Cassani, Existence and non-existence of solitary waves for the critical Klein-Gordon equation coupled with Maxwell's equations, Nonlinear Anal., to appear.

[7] G.M. Coclite, A multiplicity result for the nonlinear Schrödinger-Maxwell equations, Commun. Appl. Anal. 7 (2003), no. 2-3, 417-423.

[8] G.M. Coclite and V. Georgiev, Solitary Waves for Maxwell-Schrödinger equations, preprint.

[9] T. D'Aprile, Semiclassical states for the nonlinear Schrödinger equation with the electromagnetic field, NoDEA Nonlinear Differential Equations Appl., to appear.

[10] T. D'Aprile and D. Mugnai, Solitary Waves for nonlinear Klein-Gordon-Maxwell and Schrödinger-Maxwell equations, Proc. R. Soc. Edinb. Sect. A 134 (2004), 1-14.

[11] T. D'Aprile and J. Wei, On bound states concentrating on spheres for the MaxwellSchrödinger equation, preprint 2004.

[12] P. D'Avenia, Non-radially symmetric solutions of nonlinear Schrödinger equation coupled with Maxwell equations, Adv. Nonlinear Studies 2 (2002), 177-192.

[13] P. D'Avenia and L. Pisani, Nonlinear Klein-Gordon equations coupled with BornInfeld type equations, Electron. J. Differential Equations 2002 (2002), no. 26, 1-13.

[14] M.J. Esteban, V. Georgiev and E. Sere, Stationary waves of the Maxwell-Dirac and the Klein-Gordon-Dirac equations, Calc. Var. PDE. 4 (1996), 265-281.

[15] L.C. Evans, Partial Differential Equations, American Mathematical Society, Providence, Rhode Island (1998).

[16] D. Mugnai, Coupled Klein-Gordon and Born-Infeld type equations: looking for solitons, R. Soc. Lond. Proc. Ser. A 460 (2004), 1519-1528.

[17] S.I. Pohožaev, On the eigenfunctions of the equation $\Delta u+\lambda f(u)=0$, Soviet Math. Dokl. 5 (1965), 1408-1411.

[18] W.A. Strauss, Existence of solitary waves in higher dimensions, Comm. Math. Phys. 55 (1977), 149-162. 